\title{
Identification of the New In Vivo Metabolites of Ilaprazole in Rat Plasma after Oral Administration by LC-MS: In Silico Prediction of the $\mathrm{H}^{+} / \mathrm{K}^{+}$-ATPase Inhibitor
}

\author{
Guiqiu Zhang ${ }^{+}\left(\mathbb{D}\right.$, Kaijing Guo $^{\dagger}$, Pengfei Wang, Yingbo Shan and Chen Ma * \\ Institute of Materia Medica, Chinese Academy of Medical Sciences and Peking Union Medical College, \\ Beijing 100050, China; zhangguiqiu@imm.ac.cn (G.Z.); guokaijing@imm.ac.cn (K.G.); \\ pengfeiwang@imm.ac.cn (P.W.); shanyingbo@imm.ac.cn (Y.S.) \\ * Correspondence: mach@imm.ac.cn; Tel.: +86-010-63165239 \\ + These authors are equally contributed to this work.
}

check for updates

Citation: Zhang, G.; Guo, K.; Wang, P.; Shan, Y.; Ma, C. Identification of the New In Vivo Metabolites of Ilaprazole in Rat Plasma after Oral Administration by LC-MS: In Silico Prediction of the $\mathrm{H}^{+} / \mathrm{K}^{+}$-ATPase Inhibitor. Molecules 2021, 26, 459. https://doi.org/10.3390/ molecules26020459

Academic Editor:

Diego Muñoz-Torrero

Received: 28 December 2020

Accepted: 11 January 2021

Published: 16 January 2021

Publisher's Note: MDPI stays neutral with regard to jurisdictional claims in published maps and institutional affiliations.

Copyright: (c) 2021 by the authors. Licensee MDPI, Basel, Switzerland. This article is an open access article distributed under the terms and conditions of the Creative Commons Attribution (CC BY) license (https:// creativecommons.org/licenses/by/ $4.0 /)$.
Abstract: Ilaprazole is a proton pump inhibitor used to treat digestive diseases. In this study, blood samples were collected after oral administration of ilaprazole and prepared by liquid-liquid extraction. The metabolites of ilaprazole were detected by liquid chromatography-high-resolution mass spectrometry (LC-HRMS) and LC-MS ${ }^{n}$. A total of twelve in vivo metabolites were detected in rat plasma and six new metabolites of ilaprazole, including one reductive metabolite with sulfide (M3), two hydroxylated metabolites with sulfoxide (M7 and M8), and three oxidative metabolites with sulfone (M9, M11, and M12), were identified. The possible metabolic pathways of ilaprazole and the fragmentation behaviors of its metabolites were elucidated. The result of the in silico prediction indicates that all the new metabolites showed the potential ability to inhibit $\mathrm{H}^{+} / \mathrm{K}^{+}$-ATPase activity.

Keywords: ilaprazole; proton pump inhibitors; metabolite identification; bioactivity prediction; LC-MS

\section{Introduction}

Ilaprazole is a proton pump inhibitor (PPI) that was approved for the treatment of digestive diseases such as gastroesophageal reflux disease, peptic ulcers, and helicobacter pylori infections [1-6]. Ilaprazole selectively accumulates in the gastric parietal cells and then activates into sulfites and sulfonamides, which can irreversibly inhibit $\mathrm{H}^{+} / \mathrm{K}^{+}$-ATPase activity to suppress gastric acid secretion [5]. Compared with traditional PPIs, ilaprazole provides a better suppression of acid, especially during the night, which can satisfy the clinical requirements of relieving symptoms for a long time [7].

Drug metabolism is an important in vivo biotransformation process, displaying a significant role in the study of drug efficacy and safety [8]. The metabolism of ilaprazole is mainly mediated by CYP450 [9,10]. The metabolites of ilaprazole, M1 and M2, have been detected in human and rat plasma by liquid chromatography coupled with mass spectrometry (LC-MS) [11,12]. Four metabolites of ilaprazole (including M1, M4, and M5) have been detected in human urinary samples [13]. Nine metabolites of ilaprazole (including M1, M2, M4, M5, M6, and M10) have been identified in human liver microsome (HLM) incubation samples with ilaprazole [14]. As far as we know, there are few studies on the bioactivity of the metabolites of ilaprazole. It is thus significant to identify more ilaprazole metabolites and predict their bioactivities. LC-MS analytical technology is widely used in the identification of metabolites with high sensitivity $[15,16]$. The present study aims to identify in vivo metabolites of ilaprazole in rat plasma by LC-MS. Blood samples were collected after oral administration of ilaprazole. After being prepared by liquid-liquid extraction, the samples were detected via liquid chromatography-tandem high-resolution mass spectrometry (LC-HRMS/MS).The fragmentation behaviors of ilaprazole and its metabolites have been elucidated by LC-MS ${ }^{n}$ and LC-HRMS/MS. Twelve metabolites were 
identified in rat plasma and the possible metabolic pathways of ilaprazole in a rat model are described in Figure 1. The potential bioactivity of the new metabolites of ilaprazole was predicted by the Prediction of Activity Spectra for Substances (PASS) [17] software product.

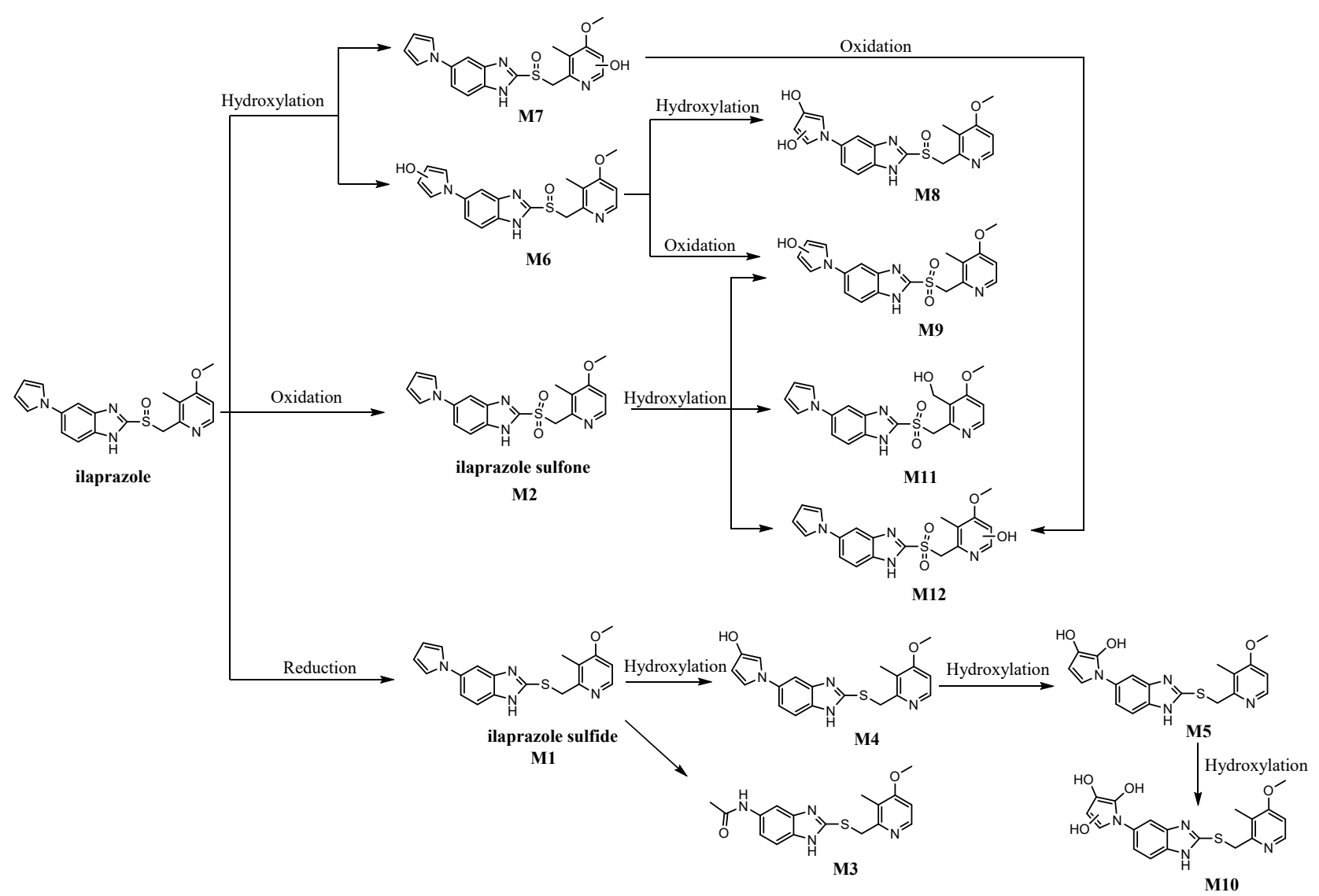

Figure 1. The metabolites of ilaprazole and the possible metabolic pathways in rats.

\section{Results and Discussion}

\subsection{Detection of Ilaprazole and Its Metabolites}

The possible metabolites of ilaprazole were determined by the following strategies. The MS/MS spectrum of ilaprazole was obtained by enhanced product ion (EPI) scanning to obtain the characteristic product ions of ilaprazole. The software LightSight was utilized to build a predictive method, the multiple reaction monitoring (MRM)-informationdependent acquisition (IDA)-EPI method, according to the product ions of ilaprazole. The characteristic fragment ion at $m / z 184$ was used as a precursor ion (Prec) -IDA-EPI mode for the Analyst 1.6 software. Some oxidative metabolites with a sulfone structure show the loss of $\mathrm{SO}_{2}(64 \mathrm{Da})$ in the fragmentation pathway [18], so the neutral loss (NL)-IDA-EPI mode of Analyst 1.6 software was selected to monitor the oxidative metabolites, including the sulfone in the structure. The HRMS/MS spectrum and the accurate mass data were obtained by LC-HRMS/MS.

Twelve metabolites were detected in the rats' plasma samples and named M1-M12. M3, M7, M8, M9, M11, and M12 are the new metabolites of ilaprazole. Their structures were characterized via high-resolution mass data. The EPI chromatograms of ilaprazole and the twelve metabolites are described in Figure 2. The accurate masses of the protonated molecule and fragment ions are shown in Supplementary Material Table S1. 


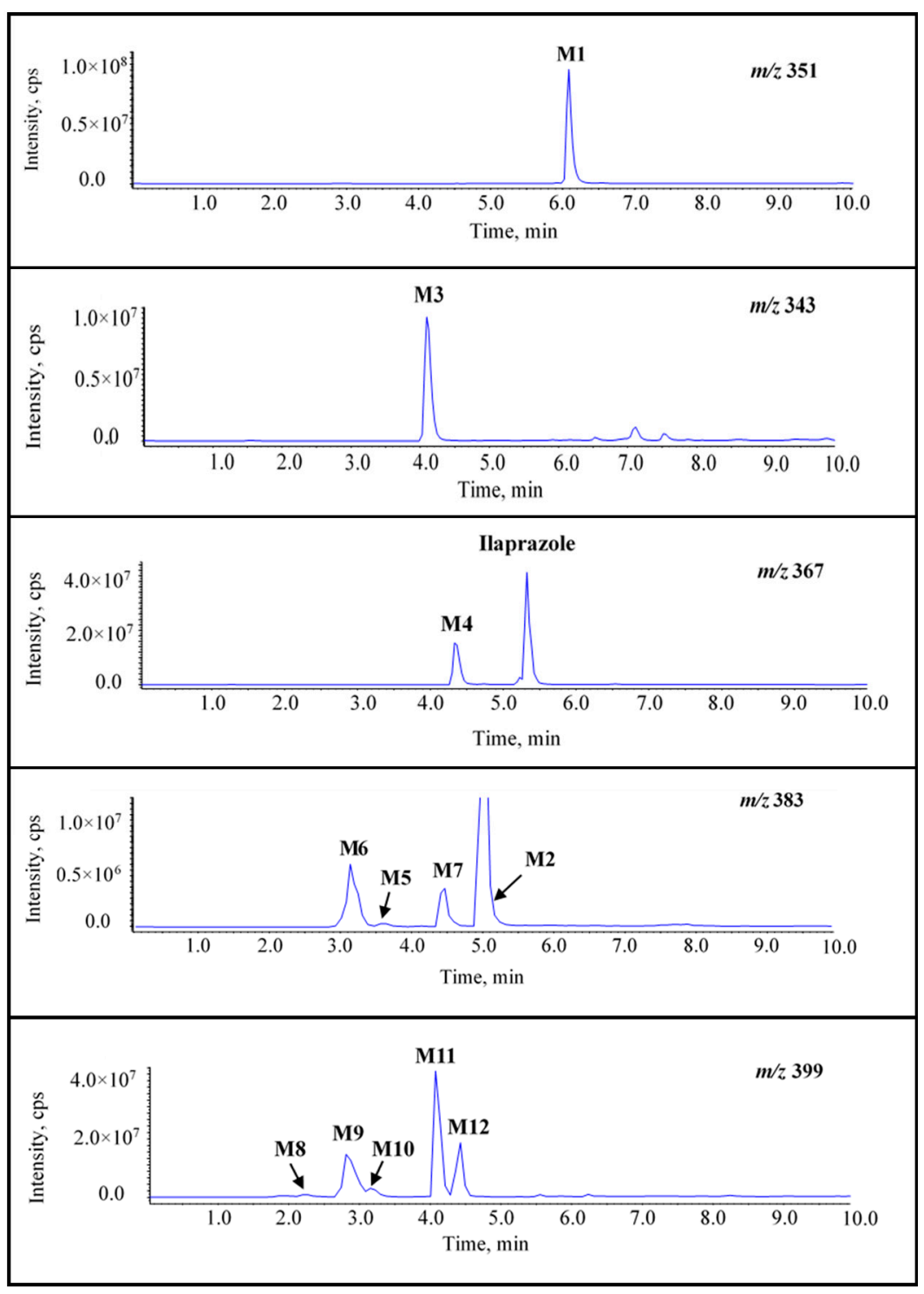

Figure 2. The enhanced product ion (EPI) chromatograms of ilaprazole and twelve metabolites.

\subsection{Characteristics of Ilaprazole, $\mathbf{M 1}$, and $\mathbf{M} \mathbf{2}$}

The metabolites of ilaprazole can be divided into three structural types, including reductive metabolites (ilaprazole sulfide derivatives), oxidative metabolites (ilaprazole sulfone derivatives), and hydroxylated metabolites (sulfoxide derivatives). The analysis of the mass spectra of ilaprazole, M1, and M2 was essential for identifying the metabolites of ilaprazole.

\subsubsection{Ilaprazole}

The $[\mathrm{M}+\mathrm{H}]^{+}$of ilaprazole was observed at $m / z 367.1220\left[\mathrm{C}_{19} \mathrm{H}_{19} \mathrm{~N}_{4} \mathrm{O}_{2} \mathrm{~S}\right]^{+}$(Supplementary Material Table S1). The dominant fragment ions at $\mathrm{m} / z 184.0865\left(\mathrm{C}_{11} \mathrm{H}_{10} \mathrm{~N}_{3}{ }^{+}\right)$and $m / z 184.0426\left(\mathrm{C}_{8} \mathrm{H}_{10} \mathrm{NO}_{2} \mathrm{~S}^{+}\right)$were formed from the fragmentation between the sulfur atom and benzimidazole [13]. Then, the ion at $m / z 184.0426$ was followed by the elimination of $\mathrm{H}_{2} \mathrm{O}$ to form the ion at $m / z$ 166. The disconnection of the bond between the C-S bond of $[\mathrm{M}+\mathrm{H}]^{+}$produced the fragment ions at $m / z 214$ and $m / z 154$ via rearrangement. The ion at $m / z 154$ was followed by the elimination of $\mathrm{H}_{2} \mathrm{O}$ to form the ion at $m / z 136$. The fragment ions at $m / z 349, m / z 137$, and $m / z 122$ were created due to the loss of $\mathrm{H}_{2} \mathrm{O}$, $\mathrm{C}_{11} \mathrm{H}_{8} \mathrm{~N}_{3} \mathrm{OS}$, and $\mathrm{C}_{12} \mathrm{H}_{11} \mathrm{~N}_{3} \mathrm{OS}$ from $[\mathrm{M}+\mathrm{H}]^{+}$, respectively. The HRMS/MS spectrum is shown in Figure 3. The $[\mathrm{M}+\mathrm{H}]^{+}$, fragment ions, and the retention time of ilaprazole were 
the same as in the reference standards. These proposed fragment ions can be supported by $\mathrm{MS}^{\mathrm{n}}$ (Supplementary Material Figure S1A). The possible fragmentation pathway of ilaprazole is depicted in Figure 3.

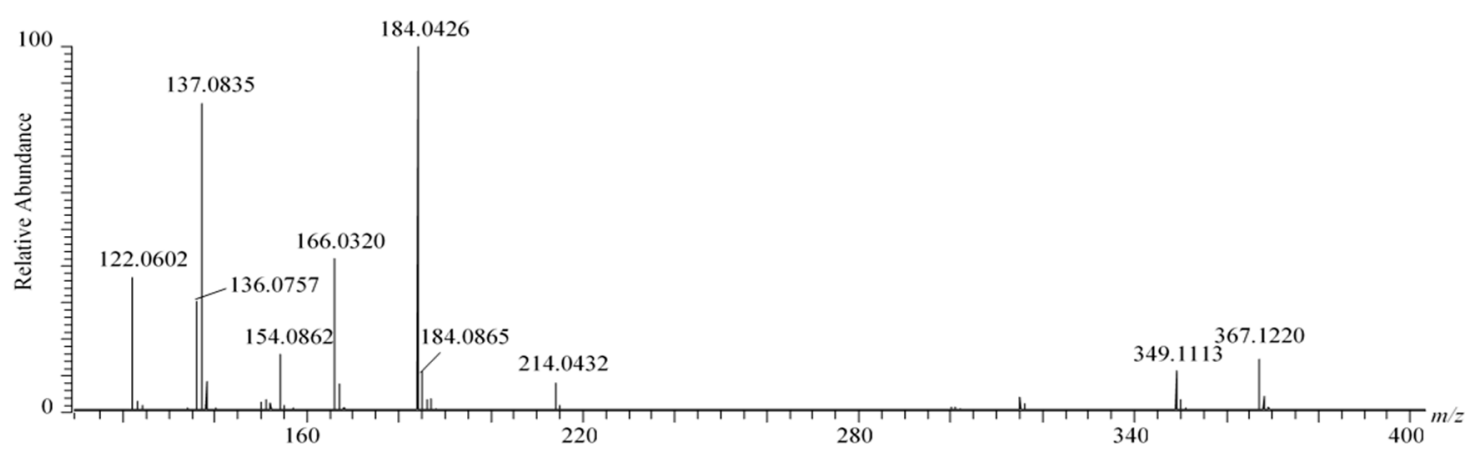<smiles>Cc1c(OCC(C)C)ccnc1CS(=O)c1nc2cc(-n3cccc3)ccc2[nH]1</smiles>

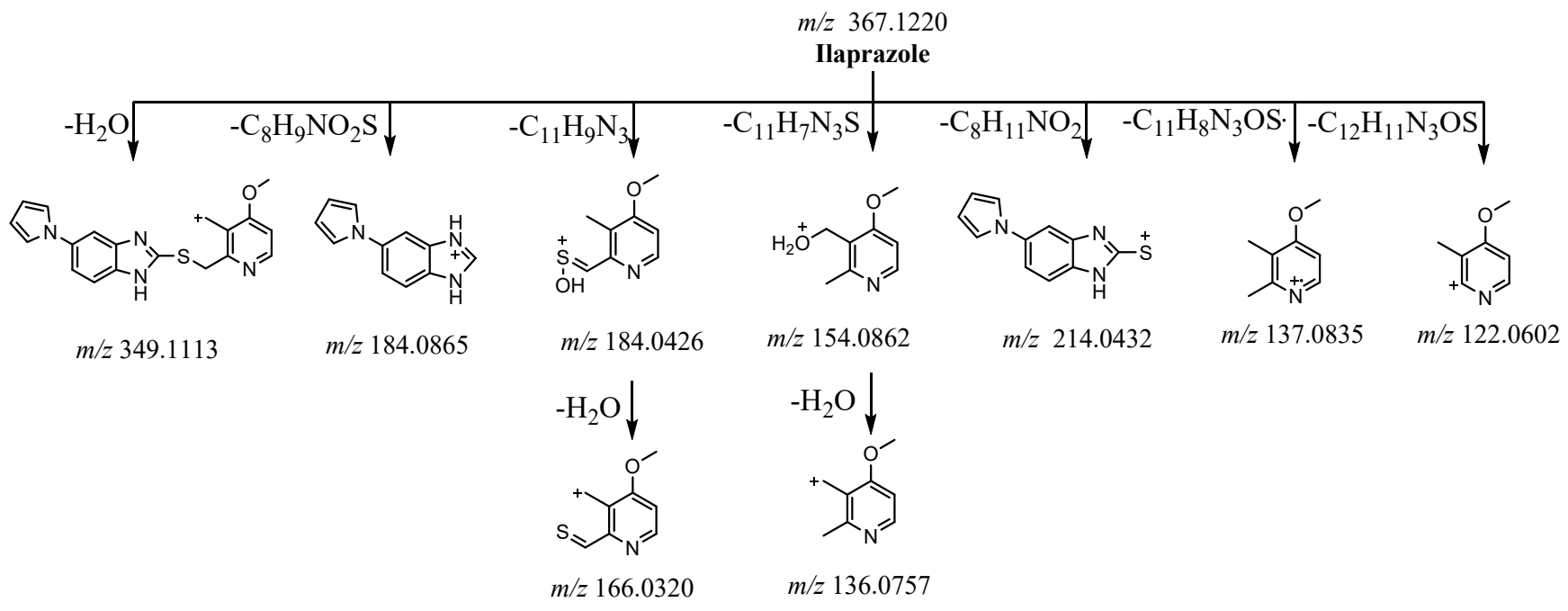

Figure 3. The tandem high-resolution mass spectrometry (HRMS/MS) spectrum and the possible fragmentation pathway of ilaprazole.

\subsubsection{M1}

The $[\mathrm{M}+\mathrm{H}]^{+}$of $\mathbf{M} 1$ was observed at $m / z 351.1270\left[\mathrm{C}_{19} \mathrm{H}_{19} \mathrm{~N}_{4} \mathrm{OS}\right]^{+}$(Supplementary Material Table S1). The protonated molecule at $m / z 351$ was 16 Da less than ilaprazole. The characteristic fragment ion at $m / z 318$ was produced to lose $33 \mathrm{Da}$ (HS) from $[\mathrm{M}+\mathrm{H}]^{+}$, which is a typical fragmentation for pyrrole-substituted benzimidazoles with a $-\mathrm{CH}_{2}-\mathrm{S}-$ link to the pyridine ring [19]. It could be deduced that the sulfoxide of ilaprazole was reduced to sulfide. The fragmentation between the sulfur atom and benzimidazole of $[\mathrm{M}+\mathrm{H}]^{+}$formed ions at $m / z 184.0869$ and $m / z$ 168.0476. The ion at $m / z 168$ was 16 Da less than $m / z 184.0426$ in ilaprazole. The ion at $m / z 168$ was followed by formation of a fragment ion at $m / z 136$ after losing S. The ions at $m / z 137$ and $m / z 122$ arose from $[\mathrm{M}+\mathrm{H}]^{+}$after losing $\mathrm{C}_{11} \mathrm{H}_{8} \mathrm{~N}_{3} \mathrm{~S}$. and $\mathrm{C}_{12} \mathrm{H}_{11} \mathrm{~N}_{3} \mathrm{~S}$, respectively. The ions at $m / z 137, m / z$ 136 , and $m / z 122$ were also observed for ilaprazole, which showed that there was the same skeletal structure in the substituted pyridine moiety. The ion at $m / z 184.0869$ was the same as ilaprazole, which suggests that there was the same skeletal structure in the substituted benzimidazole moiety. The $[\mathrm{M}+\mathrm{H}]^{+}$, fragment ions, and the retention time of $\mathbf{M 1}$ were the same as the reference standards of ilaprazole sulfide. These proposed fragment ions 
can be supported by MS ${ }^{\mathrm{n}}$ (Supplementary Material Figure S1B). Based on the above, the possible structure of M1 was deduced as ilaprazole sulfide. The HRMS/MS spectrum and the possible fragmentation pathway of $\mathbf{M 1}$ are depicted in Figure 4.

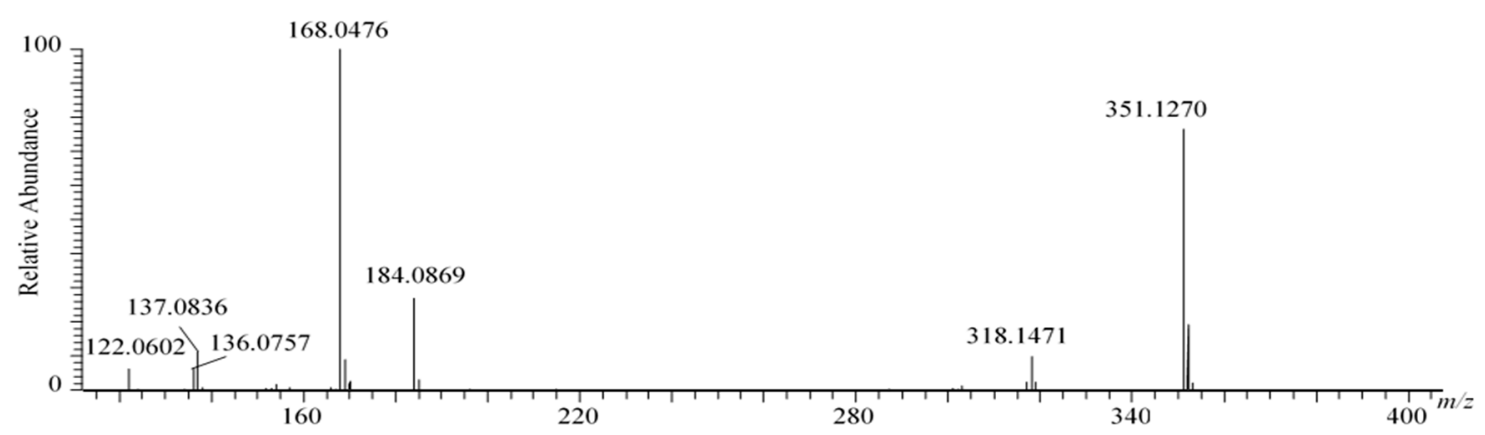<smiles>Cc1c(O[C]([In])[I+])ccnc1CSc1nc2cc(-n3cccc3)ccc2[nH]1</smiles>

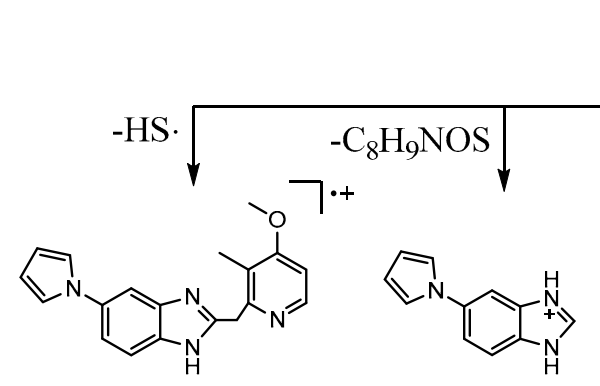

$m / z 318.1471$

$m / z 184.0869$

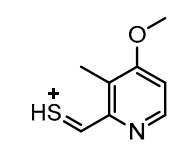

$m / z 168.0476$

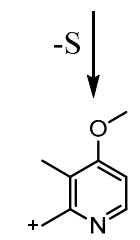

$m / z 136.0757$

Figure 4. The HRMS/MS spectrum and the possible fragmentation pathway of M1.

\subsubsection{M2}

The $[\mathrm{M}+\mathrm{H}]^{+}$of $\mathbf{M} 2$ was detected at $m / z 383.1168\left[\mathrm{C}_{19} \mathrm{H}_{19} \mathrm{~N}_{4} \mathrm{O}_{3} \mathrm{~S}\right]^{+}$(Supplementary Material Table S1). The protonated molecule at $m / z 383$ was 16 Da more than ilaprazole. The characteristic fragment ion of $\mathbf{M} 2$ at $m / z 319$ was produced to lose $64 \mathrm{Da}\left(\mathrm{SO}_{2}\right)$ from $[\mathrm{M}+\mathrm{H}]^{+}$, as suggested by the existence of sulfone [18]. The disconnection between benzimidazole and the sulfur atom of $[\mathrm{M}+\mathrm{H}]^{+}$led to the ions at $m / z 200.0376$ (16 Da more than $m / z 184.0426$ in ilaprazole) and $m / z 184.0869$ (same as ilaprazole). The ion at $m / z 200$ was followed by the elimination of $\mathrm{SO}$ and $\mathrm{SO}_{2}$ to yield the ions at $m / z 152$ and $m / z 136$, respectively. The ions at $m / z 230, m / z 137$, and $m / z 122$ arose from $[\mathrm{M}+\mathrm{H}]^{+}$after losing $\mathrm{C}_{8} \mathrm{H}_{11} \mathrm{NO}_{2}, \mathrm{C}_{11} \mathrm{H}_{8} \mathrm{~N}_{3} \mathrm{O}_{2} \mathrm{~S} \cdot$, and $\mathrm{C}_{12} \mathrm{H}_{11} \mathrm{~N}_{3} \mathrm{O}_{2} \mathrm{~S}$, respectively. The ions at $m / z 137, m / z 136$, and $m / z 122$ were also observed for ilaprazole, which suggested that there were no changes in the substituted pyridine ring. The ion at $m / z 184.0869$ was similar to ilaprazole, which suggests that there was the same skeletal structure in the substituted benzimidazole moiety. The HRMS/MS spectrum is shown in Figure 5. The $[\mathrm{M}+\mathrm{H}]^{+}$, fragment ions, and the retention time of $\mathbf{M} 2$ were the same as the reference standards of ilaprazole sulfone. These proposed fragment ions can be supported by MS $^{n}$ (Supplementary Material Figure S1C). 
Based on the above, the possible structure of M2 was deduced as ilaprazole sulfone. The possible fragmentation pathway of $\mathbf{M} 2$ is also depicted in Figure 5.

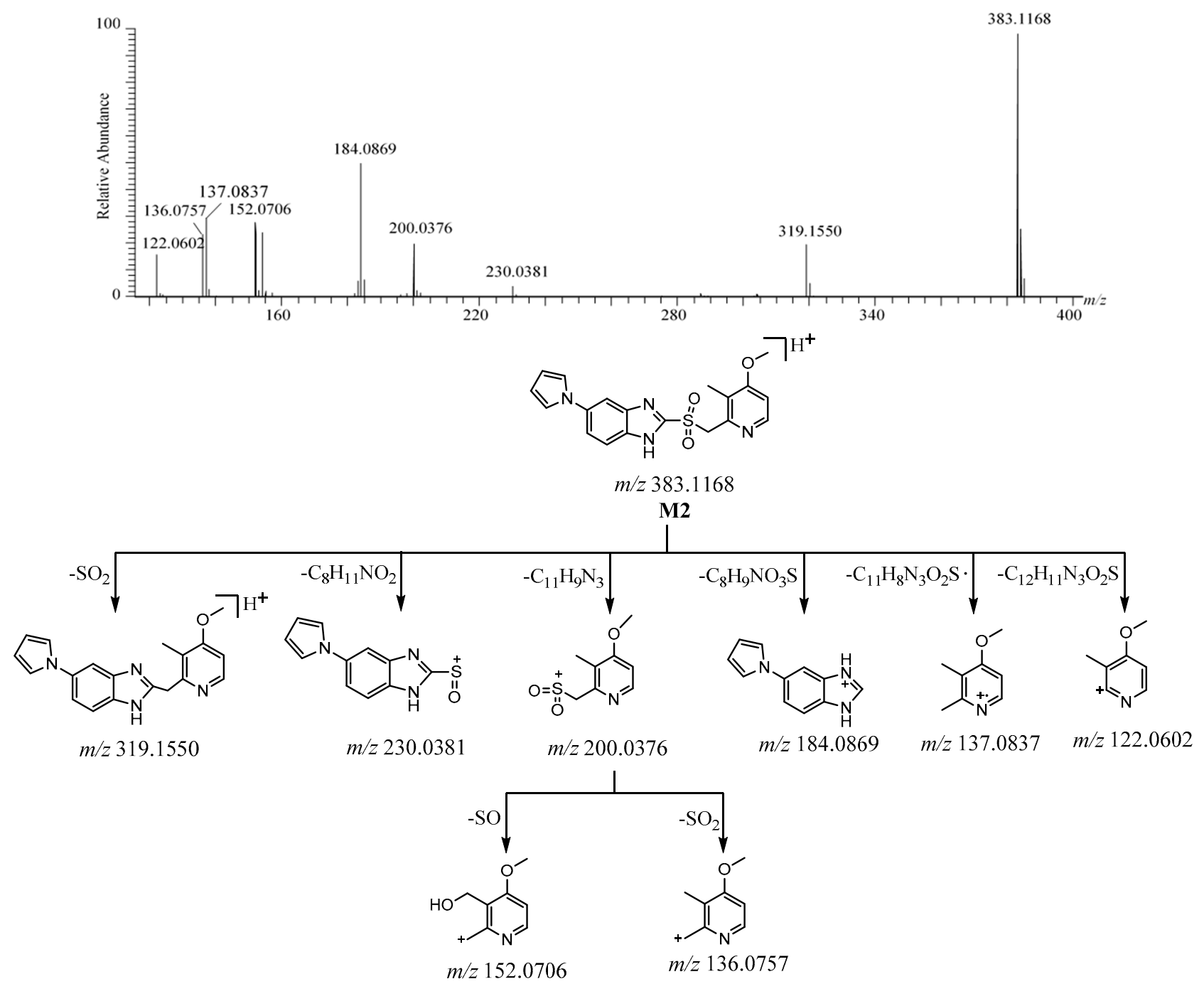

Figure 5. The HRMS/MS spectrum and the possible fragmentation pathway of M2.

\subsection{Identification of the New Metabolites of Ilaprazole}

\subsubsection{M3}

The $[\mathrm{M}+\mathrm{H}]^{+}$of $\mathbf{M} 3$ was observed at $m / z 343.1215\left[\mathrm{C}_{17} \mathrm{H}_{19} \mathrm{~N}_{4} \mathrm{O}_{2} \mathrm{~S}\right]^{+}$(Supplementary Material Table S1). The protonated molecule at $m / z 343$ was 24 Da less than ilaprazole. The characteristic fragment ion at $m / z 310$ was formed to lose $33 \mathrm{Da}(\mathrm{HS})$ from $[\mathrm{M}+\mathrm{H}]^{+}$, which indicated the existence of a sulfide bond, as with M1. The dominant fragment ions at $m / z 176.0815\left(\mathrm{C}_{9} \mathrm{H}_{10} \mathrm{~N}_{3} \mathrm{O}^{+}\right)$and $m / z 168.0474\left(\mathrm{C}_{8} \mathrm{H}_{10} \mathrm{NOS}^{+}\right)$formed as a result of the fragmentation between the sulfur atom and benzimidazole of $[\mathrm{M}+\mathrm{H}]^{+}$. The fragment ion at $m / z 176$ was followed by the production of the ion at $m / z 134$ by the elimination of $42 \mathrm{Da}\left(\mathrm{C}_{2} \mathrm{H}_{2} \mathrm{O}\right)$. It could be deduced that there may be an amide bond in $\mathbf{M} 3$. The fragment ion at $m / z 168$ was followed by losing $S$ to form a fragment ion at $m / z 136$. The ions at $m / z 137$ and $m / z 122$ arose from $[\mathrm{M}+\mathrm{H}]^{+}$after losing $\mathrm{C}_{9} \mathrm{H}_{8} \mathrm{~N}_{3} \mathrm{OS}$. and $\mathrm{C}_{10} \mathrm{H}_{11} \mathrm{~N}_{3} \mathrm{OS}$, respectively. The fragment ions at $m / z 168, m / z 137, m / z 136$, and $m / z 122$ were also observed for M1, as there were no changes in the substituted pyridine ring. The HRMS/MS spectrum is described in Figure 6. These proposed fragment ions can be supported by MS ${ }^{\mathrm{n}}$ (Supplementary Material Figure S1D). Based on the above, the possible structure and the possible fragmentation pathway of $\mathbf{M} 3$ are depicted in Figure 6. 

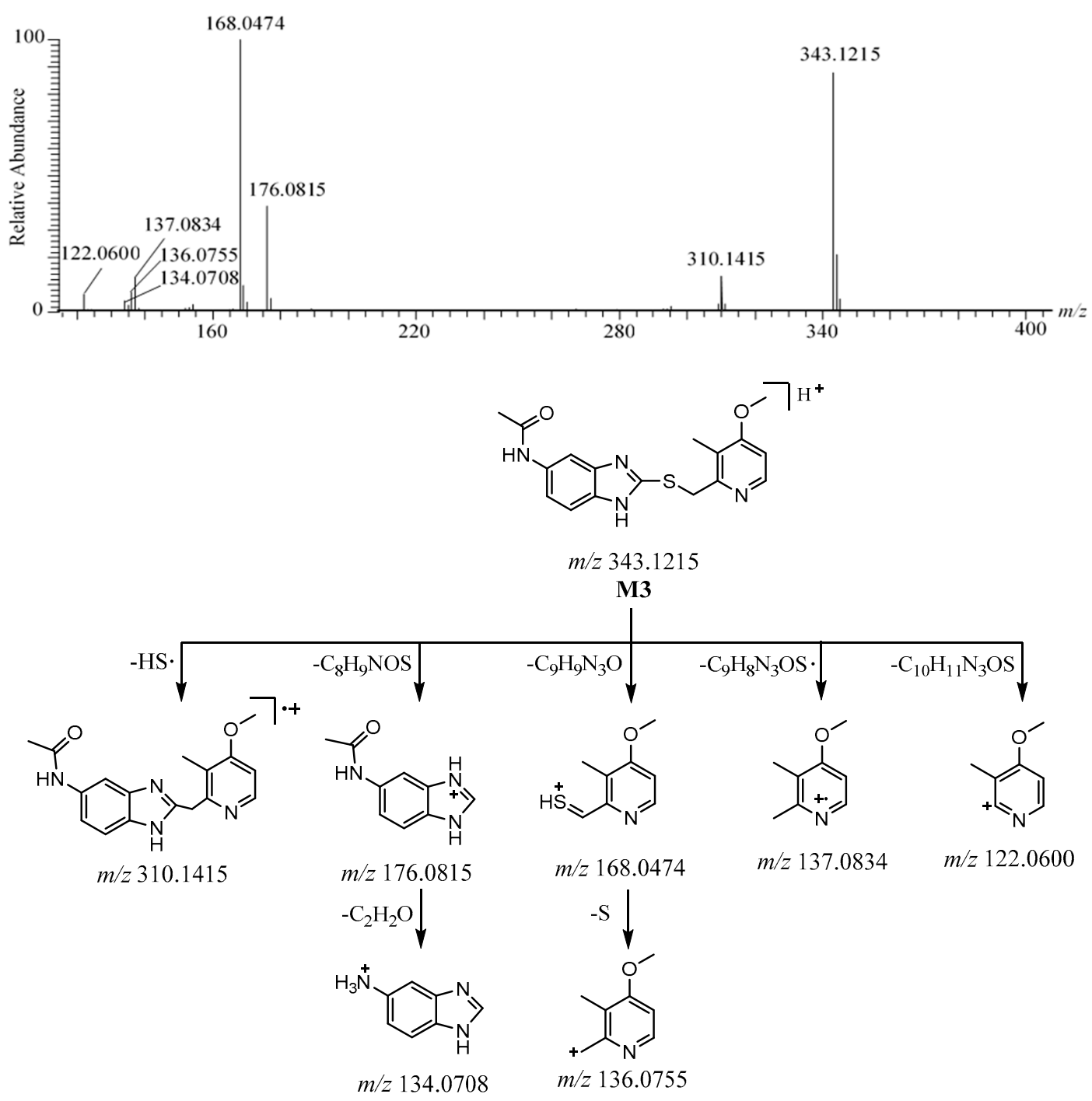

Figure 6. The HRMS/MS spectrum and the possible fragmentation pathway of M3.

\subsubsection{M7}

The $[\mathrm{M}+\mathrm{H}]^{+}$of $\mathbf{M 7}$ was observed at $m / z 383.1172\left[\mathrm{C}_{19} \mathrm{H}_{19} \mathrm{~N}_{4} \mathrm{O}_{3} \mathrm{~S}\right]^{+}$(Supplementary Material Table S1). The protonated molecule of $\mathbf{M 7}$ was 16 Da more than ilaprazole. The ions at $m / z 200.0376\left(\mathrm{C}_{8} \mathrm{H}_{10} \mathrm{NO}_{3} \mathrm{~S}^{+}\right)$and $m / z 184.0868\left(\mathrm{C}_{11} \mathrm{H}_{10} \mathrm{~N}_{3}{ }^{+}\right)$are believed to be the products of the fragmentation between the sulfur atom and benzimidazole; the ion at $m / z 200$ was followed by the elimination of $\mathrm{H}_{2} \mathrm{O}$ to form the ion at $m / z 182$, which lost $\mathrm{CO}$ to yield the ion at $m / z$ 154. The disconnection of the bond between the C-S bond of $[\mathrm{M}+\mathrm{H}]^{+}$produced the fragment ions at $m / z 214$ and $m / z 170$ via rearrangement, and the ion at $m / z 170$ was followed by the loss of $\mathrm{H}_{2} \mathrm{O}$ and $\mathrm{CO}+\mathrm{H}_{2} \mathrm{O}$ to form the ions at $m / z 152$ and $m / z 124$, respectively. This suggests that a hydroxyl group was added in the pyrrole-substituted benzimidazole moiety. The fragment ions at $\mathrm{m} / z 153$ and $\mathrm{m} / z 138$ arose from $[\mathrm{M}+\mathrm{H}]^{+}$after losing $\mathrm{C}_{11} \mathrm{H}_{8} \mathrm{~N}_{3} \mathrm{OS}$. and $\mathrm{C}_{12} \mathrm{H}_{11} \mathrm{~N}_{3} \mathrm{OS}$, respectively. The produced ions at $m / z 214$ and $m / z 184.0869$ were the same as ilaprazole, which suggests that there are no changes in the substituted benzimidazole moiety. The fragment ions at $m / z 200$, $m / z 182, m / z 170, m / z 152, m / z 153$, and $m / z 138$ were 16 Da more than those ions at $m / z$ 184.0426, $m / z 166, m / z 154, m / z 136, m / z 137$, and $m / z 122$ of ilaprazole, respectively, which indicates that a hydroxyl group was added in the pyridine moiety, and the position of the hydroxyl group could not be determined. These proposed fragment ions can be supported by MSn (Supplementary Material Figure S1H). The HRMS/MS spectrum and the possible fragmentation pathway of $\mathbf{M 7}$ are depicted in Figure 7. 

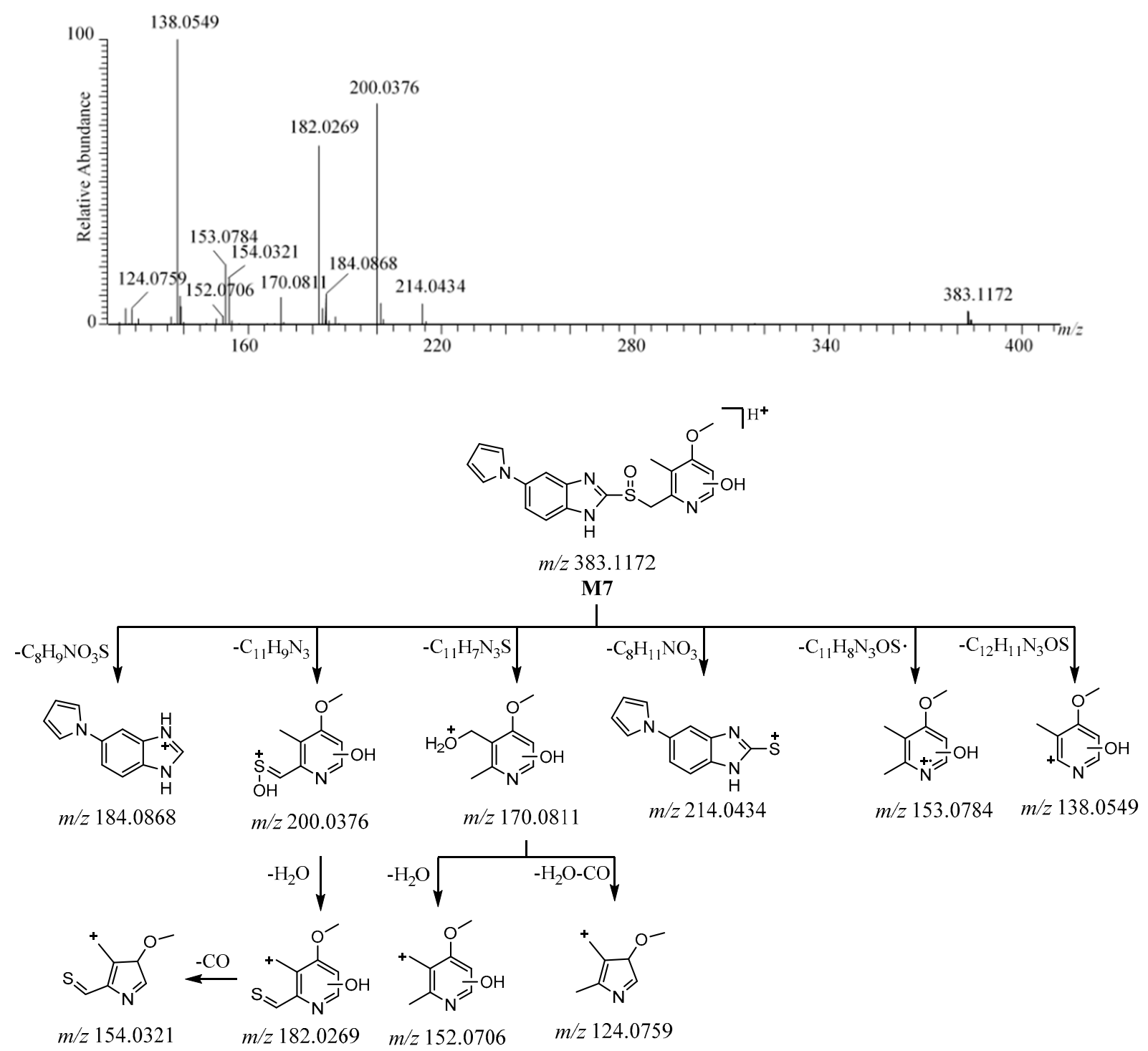

Figure 7. The HRMS/MS spectrum and the possible fragmentation pathway of M7.

\subsubsection{M8}

The $[\mathrm{M}+\mathrm{H}]^{+}$of $\mathrm{M} 8$ was detected at $m / z 399.1114\left[\mathrm{C}_{19} \mathrm{H}_{19} \mathrm{~N}_{4} \mathrm{O}_{4} \mathrm{~S}\right]^{+}$(Supplementary Material Table S1). The protonated molecule of M8 was 32 Da more than ilaprazole. The ions at $m / z 216.0761\left(\mathrm{C}_{11} \mathrm{H}_{10} \mathrm{~N}_{3} \mathrm{O}_{2}{ }^{+}\right)$and $m / z 184.0428\left(\mathrm{C}_{8} \mathrm{H}_{10} \mathrm{NO}_{2} \mathrm{~S}^{+}\right)$are believed to be the products of the fragmentation between the sulfur atom and benzimidazole, and the ion at $m / z 184.0428$ was followed by the elimination of $\mathrm{H}_{2} \mathrm{O}$ to form the ion at $\mathrm{m} / z 166$. The disconnection of the bond between the C-S bond of $[\mathrm{M}+\mathrm{H}]^{+}$produced the fragment ions at $m / z 246$ and $m / z 154$ via rearrangement, and the ion at $m / z 154$ was followed by the elimination of $\mathrm{H}_{2} \mathrm{O}$ to form the ion at $m / z$ 136. The fragment ions at $m / z 381, m / z 200$, $m / z 137$, and $m / z 122$ arose from $[\mathrm{M}+\mathrm{H}]^{+}$after losing $\mathrm{H}_{2} \mathrm{O}, \mathrm{C}_{8} \mathrm{H}_{9} \mathrm{NO}_{3} \mathrm{~S}, \mathrm{C}_{11} \mathrm{H}_{8} \mathrm{~N}_{3} \mathrm{O}_{3} \mathrm{~S}$., and $\mathrm{C}_{12} \mathrm{H}_{11} \mathrm{~N}_{3} \mathrm{O}_{3} \mathrm{~S}$, respectively. After losing $\mathrm{CO}$ from $m / z 200$, the ion at $m / z 172$ was created. The fragment ions at $\mathrm{m} / z 246$ and $\mathrm{m} / \mathrm{z} 216$ were $32 \mathrm{Da}$ more than those ions at $m / z 214$ and $m / z 184.0865$ of ilaprazole, respectively. The ion at $m / z 216$ lost $\mathrm{H}_{2} \mathrm{O}$ and CO to yield the ions at $m / z 198$ and $m / z 188$ respectively, which suggests that two hydroxyl groups were added in the pyrrole-substituted benzimidazole moiety, and the positions of the hydroxyl groups could not be determined. The fragment ions at $m / z 184.0428$, $m / z 137, m / z 136$, and $m / z 122$ were also observed for ilaprazole, as there are no changes in the substituted pyridine moiety. These proposed fragment ions can be supported by 
MS $^{n}$ (Supplementary Material Figure S1I). The HRMS/MS spectrum and the possible fragmentation pathway of M8 are described in Figure 8.
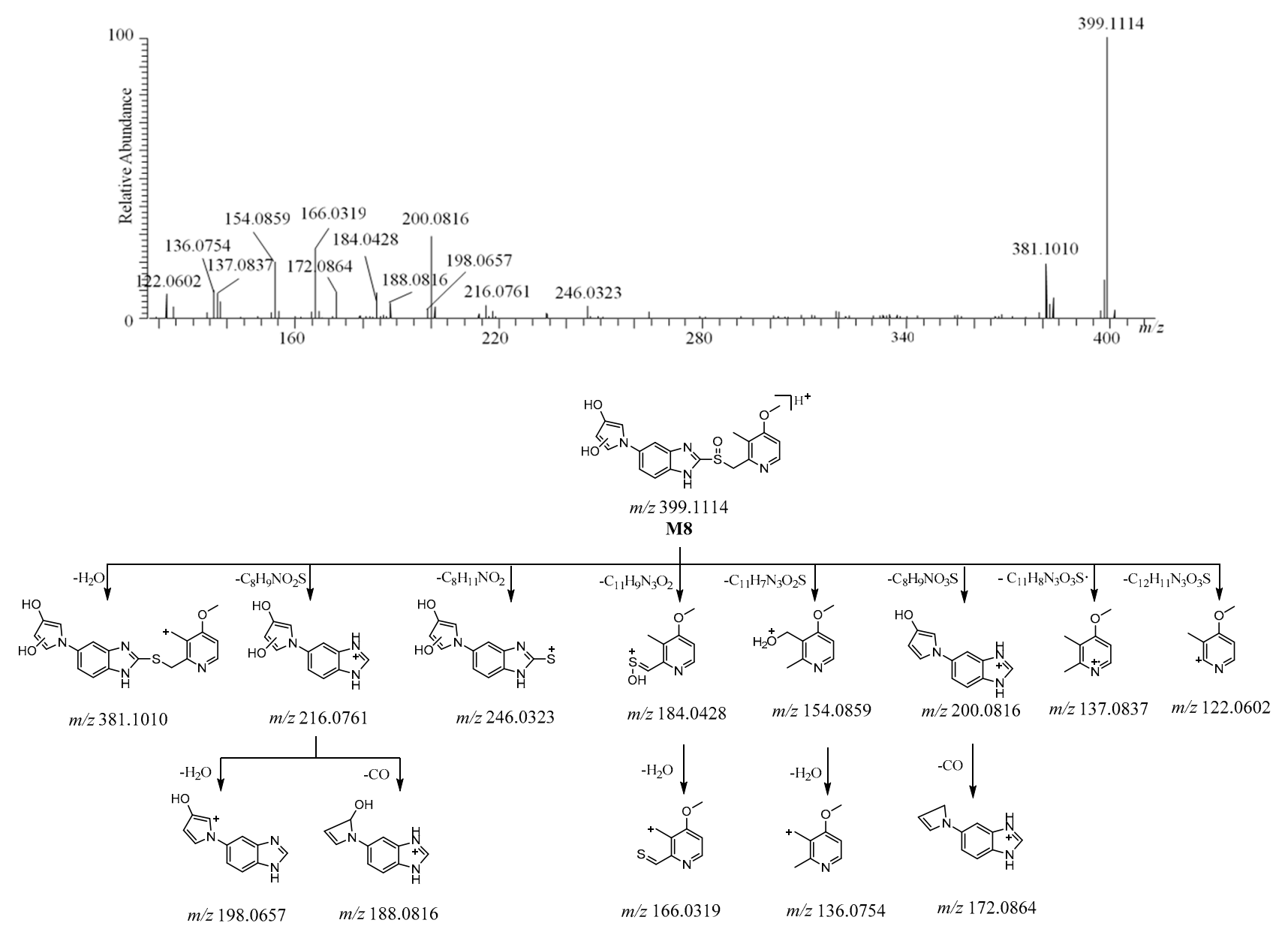

Figure 8. The HRMS/MS spectrum and the possible fragmentation pathway of M8.

\subsubsection{M9}

The $[\mathrm{M}+\mathrm{H}]^{+}$of M9 was detected at $m / z 399.1118\left[\mathrm{C}_{19} \mathrm{H}_{19} \mathrm{~N}_{4} \mathrm{O}_{4} \mathrm{~S}\right]^{+}$(Supplementary Material Table S1). The protonated molecule of M9 was $32 \mathrm{Da}$ more than ilaprazole. The characteristic fragment ion of $\mathbf{M 9}$ at $m / z 335$ was produced to lose $64 \mathrm{Da}\left(\mathrm{SO}_{2}\right)$ from $[\mathrm{M}+\mathrm{H}]^{+}$, which suggests the existence of a sulfone, as with $\mathbf{M} 2$. Then, the ion at $m / z 335$ lost $\mathrm{CO}$ to yield the ion at $\mathrm{m} / \mathrm{z}$ 307. The ions at $\mathrm{m} / \mathrm{z} 200.0817$ and $\mathrm{m} / \mathrm{z} 200.0379$ are believed to be the product ions from the fragmentation between the sulfur atom and benzimidazole of $[\mathrm{M}+\mathrm{H}]^{+}$, and the ion at $m / z 200.0379$ was followed by the elimination of SO and $\mathrm{SO}_{2}$ to form the ions at $m / z 152$ and $m / z$ 136, respectively. The ion at $m / z 172$ arose from the fragment ion at $m / z 200.0817$ after losing CO. The fragment ions at $m / z 246$, $m / z 137$, and $m / z 122$ arose from $[\mathrm{M}+\mathrm{H}]^{+}$after losing $\mathrm{C}_{8} \mathrm{H}_{11} \mathrm{NO}_{2}, \mathrm{C}_{11} \mathrm{H}_{8} \mathrm{~N}_{3} \mathrm{O}_{3} \mathrm{~S} \cdot$, and $\mathrm{C}_{12} \mathrm{H}_{11} \mathrm{~N}_{3} \mathrm{O}_{3} \mathrm{~S}$, respectively. The product ions at $\mathrm{m} / z 246$ and $\mathrm{m} / z 200.0817$ were $16 \mathrm{Da}$ more than those ions at $m / z 230$ and $m / z 184.0865$ of $\mathbf{M} 2$, respectively. A subsequent CO loss from $m / z 335$ and $m / z 200$ led to the ions at $m / z 307$ and $m / z$ 172, respectively, which could be due to the presence of hydroxyl groups in the pyrrole-substituted benzimidazole moiety, and the positions of the hydroxyl groups could not be determined. The fragment ions at $m / z 200.0379, m / z 152, m / z 137, m / z 136$, and $m / z 122$ were also observed for M2, as they may share the same skeletal structure in the substituted pyridine moiety. These proposed fragment ions can be supported by $\mathrm{MS}^{\mathrm{n}}$ (Supplementary Material Figure S1J). The HRMS/MS spectrum and the possible fragmentation pathway of M9 are described in Figure 9. 

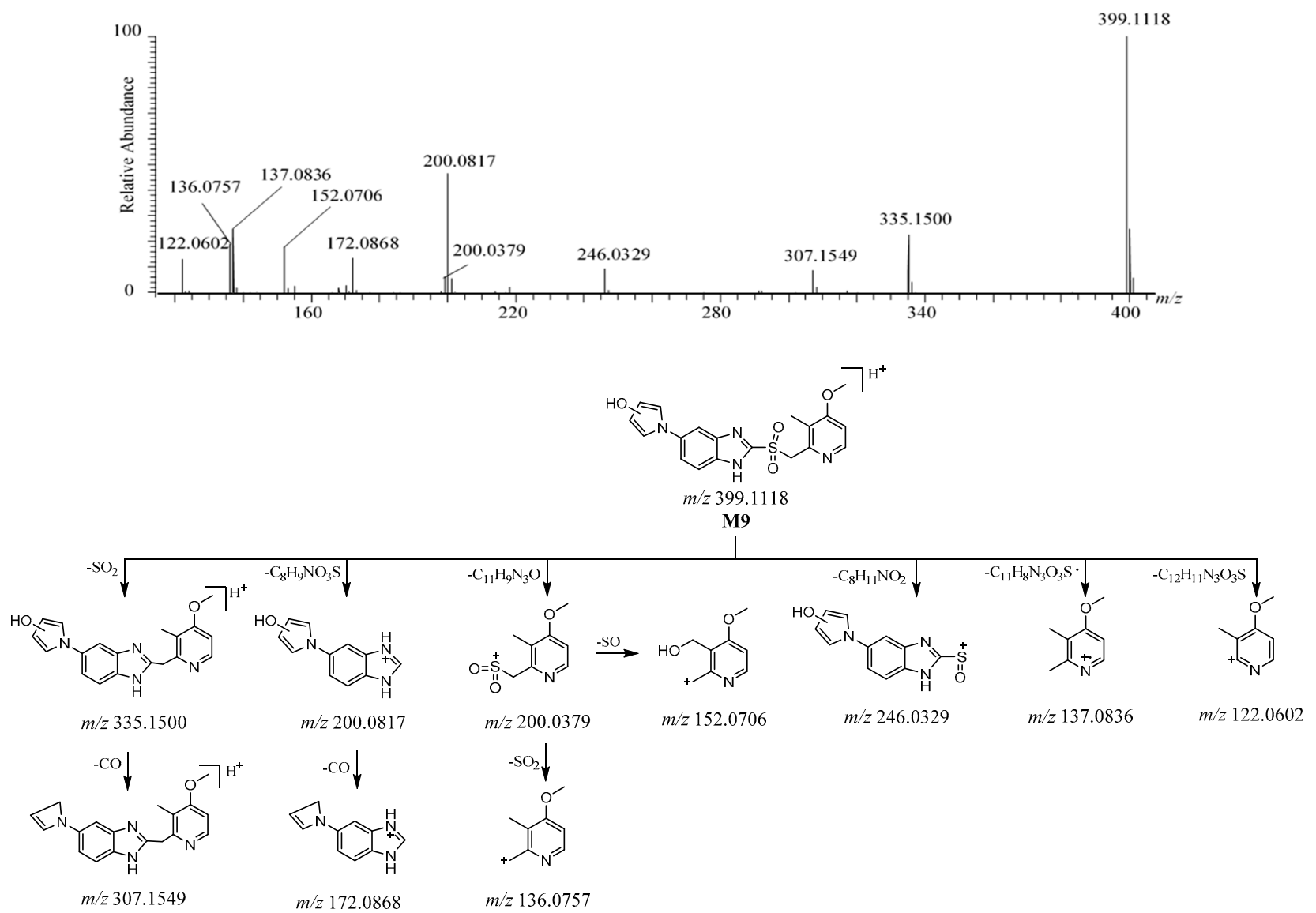

Figure 9. The HRMS/MS spectrum and the possible fragmentation pathway of M9.

\subsubsection{M11}

The $[\mathrm{M}+\mathrm{H}]^{+}$of $\mathbf{M 1 1}$ was detected at $m / z 399.1116\left[\mathrm{C}_{19} \mathrm{H}_{19} \mathrm{~N}_{4} \mathrm{O}_{4} \mathrm{~S}\right]^{+}$(Supplementary Material Table S1). The protonated molecule of M11 was 32 Da more than ilaprazole. The ion at $\mathrm{m} / z$ at 381 formed as a result of the loss of $\mathrm{H}_{2} \mathrm{O}$ from $[\mathrm{M}+\mathrm{H}]^{+}$, followed by the loss of $\mathrm{SO}_{2}$ to form the ion at $m / z 317$, which suggested the existence of sulfone, as with M2. The ions at $m / z 184.0869$ and $m / z 216.0326$ are believed to be the product ions of the fragmentation between the sulfur atom and benzimidazole. The ions at $m / z 301$ and $m / z 138$ came from $[\mathrm{M}+\mathrm{H}]^{+}$after the loss of $\mathrm{CH}_{6} \mathrm{O}_{3} \mathrm{~S}$ and $\mathrm{C}_{12} \mathrm{H}_{11} \mathrm{~N}_{3} \mathrm{O}_{2} \mathrm{~S}$, respectively. The ions at $\mathrm{m} / z 216$ and $m / z 138$ were 16 Da more than those ions at $m / z 200$ and $m / z 122$ in M2, suggesting that a hydroxyl group was added in the pyridine moiety. The ion at $m / z 184.0868$ was also observed for M2, which shows that there was the same skeletal structure in the pyrrole-substituted benzimidazole moiety. These proposed fragment ions can be supported by MS ${ }^{n}$ (Supplementary Material Figure S1L). Based on the above, the HRMS/MS spectrum, the possible structure, and the possible fragmentation pathway of M11 are depicted in Figure 10. 

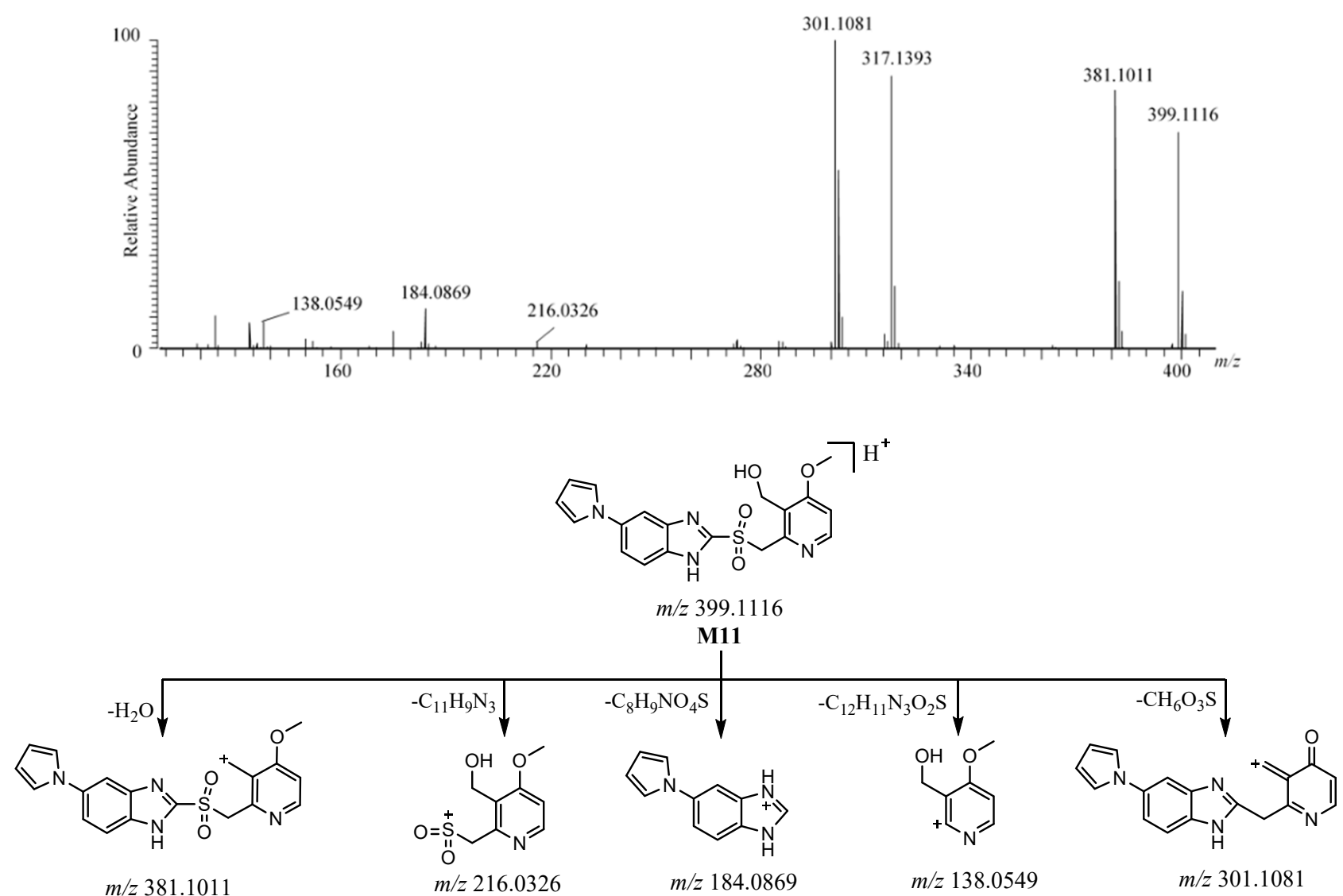<smiles>COc1ccnc(Cc2nc3cc(-n4cccc4)ccc3[nH]2)c1C</smiles>

$m / z 317.1393$

Figure 10. The HRMS/MS spectrum and the possible fragmentation pathway of M11.

\subsubsection{M12}

The $[\mathrm{M}+\mathrm{H}]^{+}$of $\mathbf{M 1 2}$ was detected at $m / z 399.1118\left[\mathrm{C}_{19} \mathrm{H}_{19} \mathrm{~N}_{4} \mathrm{O}_{4} \mathrm{~S}\right]^{+}$(Supplementary Material Table S1). The protonated molecule of M12 was 32 Da more than ilaprazole. The ions at $m / z 184.0868$ and $m / z 216.0324$ are believed to be the product ions of the fragmentation between the sulfur atom and benzimidazole. The ion at $m / z 152$ came from the ion at $m / z 216$ after the loss of $\mathrm{SO}_{2}$, which suggests the existence of sulfone, as with $\mathbf{M} 2$. The ion at $m / z 140$ came from $[\mathrm{M}+\mathrm{H}]^{+}$after the loss of $\mathrm{C}_{12} \mathrm{H}_{9} \mathrm{~N}_{3} \mathrm{O}_{2} \mathrm{~S}$. The fragment ion at $m / z 184.0868$ was also observed for $\mathbf{M} 2$, which shows that there was the same skeletal structure in the pyrrole-substituted benzimidazole moiety. The fragment ions at $m / z 216$ and $m / z 152$ were 16 Da more than those ions at $m / z 200$ and $m / z 136$ in M2, which shows that there was a hydroxyl group in the pyridine moiety, and the position of the hydroxyl group could not be determined. These proposed fragment ions can be supported by MS $^{n}$ (Supplementary Material Figure S1M). The HRMS/MS spectrum and the possible fragmentation pathway of M12 are described in Figure 11. 

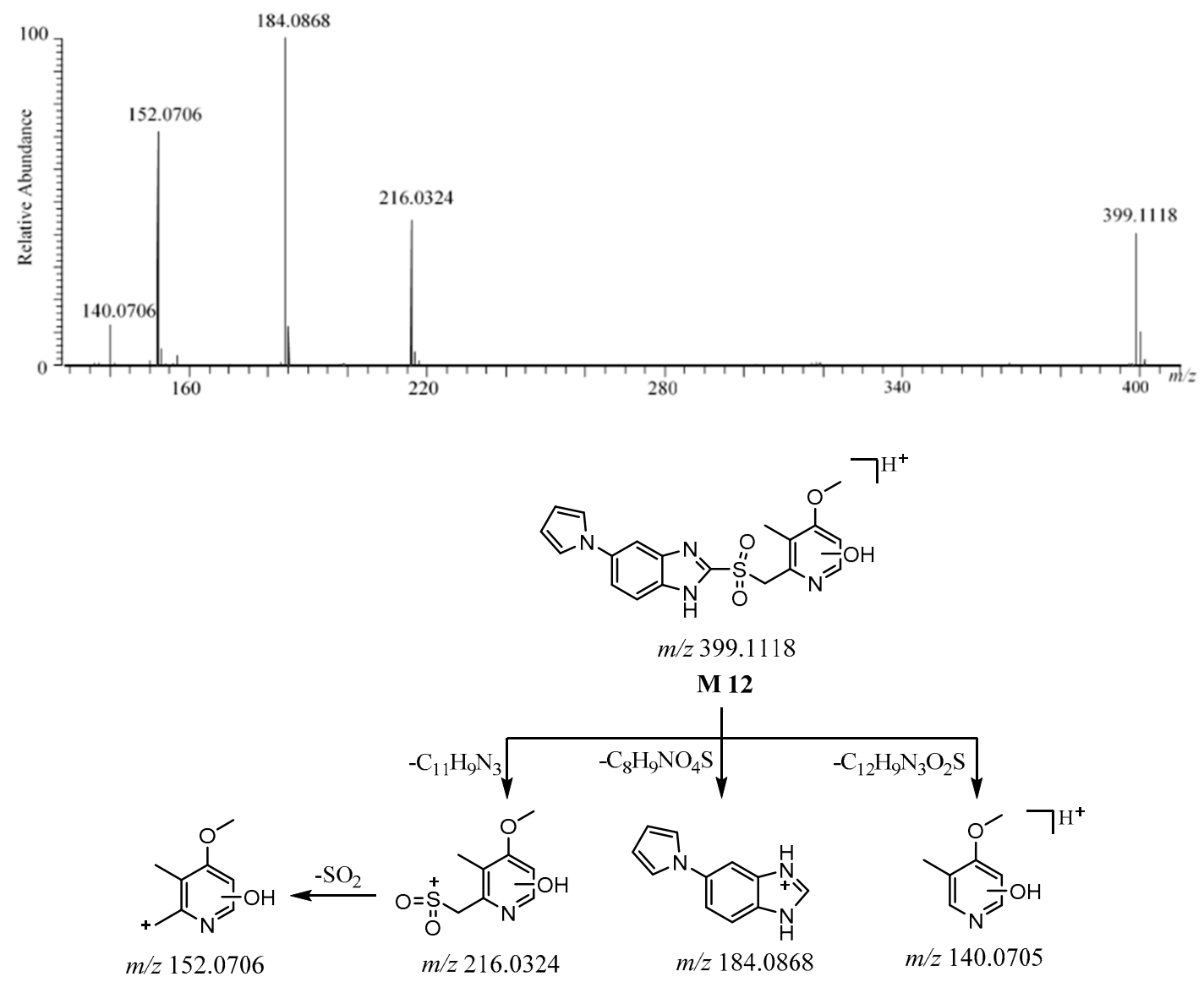

Figure 11. The HRMS/MS spectrum and the possible fragmentation pathway of M12.

\subsection{Identification of the Known Metabolites, M4, M5, M6, and M10}

\subsubsection{M4}

The $[\mathrm{M}+\mathrm{H}]^{+}$of $\mathrm{M} 4$ was observed at $m / z 367.1219\left[\mathrm{C}_{19} \mathrm{H}_{19} \mathrm{~N}_{4} \mathrm{O}_{2} \mathrm{~S}\right]^{+}$(Supplementary Material Table S1). The protonated molecule at $m / z 367$ was the same as ilaprazole. The HRMS/MS spectrum of M4 (Supplementary Material Figure S2A) showed product ions at $m / z 334$ (loss of HS.), $m / z 200$ (loss of $\mathrm{C}_{8} \mathrm{H}_{9} \mathrm{NOS}$ ), $m / z 172$ (loss of $\mathrm{C}_{8} \mathrm{H}_{9} \mathrm{NOS}+\mathrm{CO}$ ), $m / z 168$ (loss of $\mathrm{C}_{11} \mathrm{H}_{9} \mathrm{~N}_{3} \mathrm{O}$ ), $m / z 137$ (loss of $\mathrm{C}_{11} \mathrm{H}_{8} \mathrm{~N}_{3} \mathrm{OS} \cdot$ ), $m / z 136$ (loss of $\mathrm{C}_{11} \mathrm{H}_{9} \mathrm{~N}_{3} \mathrm{O}+\mathrm{S}$ ), and $m / z 122$ (loss of $\mathrm{C}_{12} \mathrm{H}_{11} \mathrm{~N}_{3} \mathrm{OS}$ ). These proposed fragment ions can be supported by MS ${ }^{\mathrm{n}}$ (Supplementary Material Figure S1E). The structure of M4 (Figure 1) was consistent with the literature [13]. The possible fragmentation pathway of M4 is described in Figure S2A.

\subsubsection{M5}

The $[\mathrm{M}+\mathrm{H}]^{+}$of $\mathbf{M} 5$ was observed at $m / z$ 383.1161 $\left[\mathrm{C}_{19} \mathrm{H}_{19} \mathrm{~N}_{4} \mathrm{O}_{3} \mathrm{~S}\right]^{+}$(Supplementary Material Table S1). The protonated molecule at $m / z 383$ was 16 Da more than ilaprazole. The HRMS/MS spectrum of M5 (Supplementary Material Figure S2B) showed product ions at $m / z 365$ (loss of $\mathrm{H}_{2} \mathrm{O}$ ), $m / z 350$ (loss of HS.), $m / z 216$ (loss of $\mathrm{C}_{8} \mathrm{H}_{9} \mathrm{NOS}$ or $\mathrm{HS}+\mathrm{C}_{8} \mathrm{H}_{8} \mathrm{NO}$ ), $m / z 188$ (loss of $\left.\mathrm{C}_{8} \mathrm{H}_{9} \mathrm{NOS}+\mathrm{CO}\right), m / z 168$ (loss of $\mathrm{C}_{11} \mathrm{H}_{9} \mathrm{~N}_{3} \mathrm{O}_{2}$ ), $m / z 137$ (loss of $\mathrm{C}_{11} \mathrm{H}_{8} \mathrm{~N}_{3} \mathrm{O}_{2} \mathrm{~S}$ ), $m / z 136$ (loss of $\mathrm{C}_{11} \mathrm{H}_{9} \mathrm{~N}_{3} \mathrm{O}_{2}+\mathrm{S}$ ), and $m / z 122$ (loss of $\mathrm{C}_{12} \mathrm{H}_{11} \mathrm{~N}_{3} \mathrm{O}_{2} \mathrm{~S}$ ). These proposed fragment ions can be supported by $\mathrm{MS}^{\mathrm{n}}$ (Supplementary Material Figure S1F). The structure of M5 (Figure 1) was consistent with the literature [13]. The possible fragmentation pathway of M5 is described in Figure S2B.

\subsubsection{M6}

The $[\mathrm{M}+\mathrm{H}]^{+}$of $\mathbf{M 6}$ was observed at $m / z 383.1169\left[\mathrm{C}_{19} \mathrm{H}_{19} \mathrm{~N}_{4} \mathrm{O}_{3} \mathrm{~S}\right]^{+}$(Supplementary Material Table S1). The protonated molecule of M6 was 16 Da more than ilapra- 
zole. The HRMS/MS spectrum of M6 (Supplementary Material Figure S2C) showed product ions at $m / z 365$ (loss of $\mathrm{H}_{2} \mathrm{O}$ ), $m / z 230$ (loss of $\mathrm{C}_{8} \mathrm{H}_{11} \mathrm{NO}_{2}$ ), $m / z 200$ (loss of $\mathrm{C}_{8} \mathrm{H}_{9} \mathrm{NO}_{2} \mathrm{~S}$ ), $m / z 184$ (loss of $\mathrm{C}_{11} \mathrm{H}_{9} \mathrm{~N}_{3} \mathrm{O}$ ), $m / z 172$ (loss of $\mathrm{C}_{8} \mathrm{H}_{9} \mathrm{NO}_{2} \mathrm{~S}+\mathrm{CO}$ ), $m / z 166$ (loss of $\mathrm{C}_{11} \mathrm{H}_{9} \mathrm{~N}_{3} \mathrm{O}+\mathrm{H}_{2} \mathrm{O}$ ), $m / z 154$ (loss of $\mathrm{C}_{11} \mathrm{H}_{7} \mathrm{~N}_{3} \mathrm{OS}$ ), $m / z 137$ (loss of $\mathrm{C}_{11} \mathrm{H}_{8} \mathrm{~N}_{3} \mathrm{O}_{2} \mathrm{~S} \cdot$ ) , m/z 136 (loss of $\mathrm{C}_{11} \mathrm{H}_{7} \mathrm{~N}_{3} \mathrm{OS}+\mathrm{H}_{2} \mathrm{O}$ ), and $m / z 122$ (loss of $\mathrm{C}_{12} \mathrm{H}_{11} \mathrm{~N}_{3} \mathrm{O}_{2} \mathrm{~S}$ ). These proposed fragment ions can be supported by MS ${ }^{\mathrm{n}}$ (Supplementary Material Figure S1G). The structure of M6 (Figure 1) was consistent with the literature [19]. The possible fragmentation pathway of $\mathrm{M} 6$ is described in Figure S2C.

\subsubsection{M10}

The $[\mathrm{M}+\mathrm{H}]^{+}$of $\mathbf{M 1 0}$ was observed at $m / z 399.1115\left[\mathrm{C}_{19} \mathrm{H}_{19} \mathrm{~N}_{4} \mathrm{O}_{4} \mathrm{~S}\right]^{+}$(Supplementary Material Table S1). The protonated molecule of M10 was 32 Da more than ilaprazole. The HRMS/MS spectrum of M10 (Supplementary Material Figure S2D) showed product ions at $m / z 366$ (loss of HS.), $m / z 232$ (loss of $\mathrm{C}_{8} \mathrm{H}_{9} \mathrm{NOS}$ ), $m / z 214$ (loss of $\mathrm{C}_{8} \mathrm{H}_{9} \mathrm{NOS}+\mathrm{H}_{2} \mathrm{O}$ ), $m / z 186$ (loss of $\mathrm{C}_{8} \mathrm{H}_{9} \mathrm{NOS}+\mathrm{H}_{2} \mathrm{O}+\mathrm{CO}$ ), $m / z 168$ (loss of $\mathrm{C}_{11} \mathrm{H}_{9} \mathrm{~N}_{3} \mathrm{O}_{3}$ ), $m / z 137$ (loss of $\mathrm{C}_{11} \mathrm{H}_{8} \mathrm{~N}_{3} \mathrm{O}_{3} \mathrm{~S} \cdot$ ) , $m / z 136$ (loss of $\mathrm{C}_{11} \mathrm{H}_{9} \mathrm{~N}_{3} \mathrm{O}_{3}+\mathrm{S}$ ), $m / z 134$ (loss of $\mathrm{C}_{8} \mathrm{H}_{9} \mathrm{NOS}_{+} \mathrm{C}_{4} \mathrm{H}_{2} \mathrm{O}_{3}$ ), and $m / z 122$ (loss of $\mathrm{C}_{12} \mathrm{H}_{11} \mathrm{~N}_{3} \mathrm{O}_{3} \mathrm{~S}$ ). These proposed fragment ions can be supported by MSn (Supplementary Material Figure S1K). The structure of M10 (Figure 1) was consistent with the literature [19]. The possible fragmentation pathway of M10 is described in Figure S2D.

\subsection{Metabolic Pathways}

CYP450 is involved in the metabolism of ilaprazole. The reaction in which sulfoxide oxidizes to sulfone was mainly mediated by CYP3A $[9,10]$. Twelve metabolites of ilaprazole were detected in rats after oral administration. The possible metabolic pathways are summarized as follows and are depicted in Figure 1. The ilaprazole sulfoxide reduction to sulfide formed M1, followed by oxidation to form M3, M4, M5, and M10. The ilaprazole sulfoxide oxidation to sulfone created M2 and subsequently hydroxylated to form M9, M11, and M12. The hydroxylation reaction of ilaprazole produced M6, M7, and M8.

\subsection{In Silico Bioactivity Prediction of the $\mathrm{H}^{+} / \mathrm{K}^{+}$-ATPase Inhibitor}

The potential bioactivities of the six new in vivo metabolites of ilaprazole were predicted. All of them have shown the potential ability to inhibit $\mathrm{H}^{+} / \mathrm{K}^{+}$-ATPase activity (Table 1).

Table 1. In silico bioactivity prediction of the new metabolites.

\begin{tabular}{cccc}
\hline Name & Probability of $\mathbf{H}^{+} / \mathbf{K}^{+}$-ATPase Inhibitor & Name & Probability of $\mathbf{H}^{+} / \mathbf{K}^{+}$-ATPase Inhibitor \\
\hline Ilaprazole & $94.1 \%$ & M8-3 & $68.4 \%$ \\
M3 & $60.9 \%$ & M9-1 & $88.2 \%$ \\
M7-1 * & $89.4 \%$ & M9-2 & $73.9 \%$ \\
M7-2 & $76.5 \%$ & M11 & $84.3 \%$ \\
M8-1 $^{*}$ & $79.1 \%$ & $\mathbf{M 1 2 - 1} *$ & $84.0 \%$ \\
M8-2 $^{*}$ & $90.7 \%$ & $\mathbf{M 1 2 - 2}{ }^{*}$ & $66.1 \%$ \\
\hline
\end{tabular}

* The possible structure is shown in Supplementary Material Table S2.

We have predicted the potential bioactivity of all the probable structures of M7, M8, M9, and M12. The probability of the bioactivity may be related to the position of the hydroxyl groups. The metabolites with a hydroxyl group in the meta-position of $\mathrm{N}$ in the pyridine moiety have shown a higher bioactivity probability than the metabolites with a hydroxyl group in the ortho-position of N such as M7-1 and M12-1. Similarly, the metabolites with a hydroxyl group in the meta-position of $\mathrm{N}$ in the pyrrole ring also displayed a higher bioactivity probability, which can be confirmed by M8-2 and M9-1. The bioactivity probability of M8-2 with two meta-hydroxyl groups was more than $90 \%$. 


\section{Materials and Methods}

\subsection{Chemicals and Reagents}

Ilaprazole ( $>99 \%)$ was purchased from the National Institutes for Food and Drug Control (Beijing, China). Ilaprazole sulfide ( $\geq 95 \%$ ) and ilaprazole sulfone ( $\geq 95 \%$ ) were purchased from Shanghai Yichun Technology Co. Ltd. (Shanghai, China). Methanol (HPLC grade) and ethyl acetate (HPLC grade) were obtained from Honeywell International Inc. (Morris, NJ, USA). Ammonium acetate (HPLC grade) was obtained from Roe Scientific Inc. (St. Newark, DE, USA). Ammonia (analytical grade) was purchased from Beijing Chemical Industry Group Co. Ltd. (Beijing, China). Heparin (analytical grade) and sodium carboxymethyl cellulose (chemical grade) were purchased from Sinopharm Chemical Reagent Co. Ltd. (Shanghai, China). Deionized water was obtained from a Mill-Q Reference water purification system (Millipore, Molsheim, France).

\subsection{Instrument and Analysis Conditions}

\subsection{1. ${\mathrm{LC}-M S^{\mathrm{n}}}^{-}$}

For separation, the processed plasma samples were treated on a chromatography system (UFLC-20AD XR, Shimadzu, Japan). The shim-pack XR-ODS II reversed-phase column (3.0 mm $\times 75 \mathrm{~mm}, 2.2 \mu \mathrm{m}$, Shimadzu, Japan) was used in this progress. The mobile phase consisted of $10 \mathrm{mM}$ ammonium acetate ( $\mathrm{pH} 7$, Solvent $\mathrm{A}$ ) and methanol (Solvent B). The gradient elution was set as follows: $0-0.5 \mathrm{~min}, 50 \% \mathrm{~B} ; 0.5-4 \mathrm{~min}, 50-85 \% \mathrm{~B} ; 4-8 \mathrm{~min}$, $85 \% \mathrm{~B} ; 8-9 \mathrm{~min}, 85-50 \% \mathrm{~B} ; 9-10 \mathrm{~min}, 50 \%$ B. The injection volume was $3 \mu \mathrm{L}$, with a flow rate of $0.4 \mathrm{~mL} / \mathrm{min}$, and the column temperature was maintained at $30^{\circ} \mathrm{C}$.

For the mass spectrometry analysis, the UFLC-20AD XR was connected to a Sciex Qtrap 5500 mass system (AB SCIEX, Framingham, MA, USA) via electrospray ionization (ESI) interface in positive ion mode and controlled by Analyst software (AB SCIEX, Framingham, MA, USA) (1.6 Version). The spectra were obtained in the EPI and $\mathrm{MS}^{3}$ scan mode. The typical parameters for MS scan were optimized as follows: ion spray voltage (IS), $5500 \mathrm{~V}$; temperature (TEM), $550{ }^{\circ} \mathrm{C}$; curtain gas (CUR), $30 \mathrm{psi}$; ion source gas 1 (GS1), $50 \mathrm{psi}$; ion source gas 2 (GS2), $35 \mathrm{psi}$; declustering potential (DP), $20 \mathrm{~V}$; entrance potential (EP), $10 \mathrm{~V}$; collision energy (CE), 25-40 V; and collision cell exit potential (CXP), $10 \mathrm{~V}$. High-purity nitrogen (99.9\%) was used to supply the MS.

\subsubsection{LC-HRMS}

The separation condition was the same as in Section 3.2.1. The extracts were separated using the Dionex Ultimate 3000 (Dionex, Germering, Germany) system and analyzed using a Q Exactive orbitrap mass spectrometer (Thermo Scientific Fisher, San Jose, CA, USA). The ESI source was operated in positive mode using a parallel reaction monitoring (PRM) scan with the following parameters. Spray voltage, $3500 \mathrm{~V}$; capillary temperature, $300^{\circ} \mathrm{C}$; sheath gas, 45 arb; aux gas, 10 arb; scan range, $m / z$ 50-850; resolution, 17500; the automatic gain control (AGC) target, $2 \times 10^{5}$; isolation window, $4.0 \mathrm{~m} / z$; (N) CE/stepped (N) CE: 10, 30, 55 V. Xcalibur software version 2.07 (Thermo Fisher Scientific, USA) was used for the data acquisition and analysis.

\subsection{Animals and Drug Administration}

Male Sprague-Dawley rats (180-220 g) were obtained from the National Institutes for Food and Drug Control (Beijing, China). Before the experiment, all the rats were bred in a controlled breeding environment. All the experimental procedures in this study were performed following the Guide for Institute of Materia Medica, Chinese Academy of Medical Science. The rats were fasted for $24 \mathrm{~h}$ with free access to water before administration. Ilaprazole suspension in 0.5\% CMC-Na was administered to the rats via gavage at a dose of $20 \mathrm{mg} / \mathrm{kg}$. 


\subsection{Pretreatment of the Sample}

Blood samples were taken from the angular vein after oral administration and put into heparinized Eppendorf tubes. Plasma samples were obtained via centrifugation at $4{ }^{\circ} \mathrm{C}$ for $10 \mathrm{~min}$ at $3500 \mathrm{r} / \mathrm{min}$. The treated samples were stored at $-80{ }^{\circ} \mathrm{C}$ until analysis. The plasma samples were then thawed at room temperature. Ethyl acetate and the plasma samples were added into a clean Eppendorf tube at a ratio of 6:1, vortexed for $5 \mathrm{~min}$, and centrifuged for $10 \mathrm{~min}$ at $8000 \mathrm{r} / \mathrm{min}$. The upper organic phase was transferred into a clean Eppendorf tube and dried with $\mathrm{N}_{2}$. The residues were reconstituted with $200 \mu \mathrm{L}$ of a $10 \mathrm{mM}$ ammonium acetate-methanol (2:8, $v / v$, adjusted to $\mathrm{pH} 9-10$ with ammonia solution) and filtered through a $0.22 \mu \mathrm{m}$ microporous membrane before the LC-MS analysis.

\subsection{In Silico Bioactivity Prediction}

PASS software can predict biochemical mechanisms and more than 300 pharmacological effects on the basis of the structural formula of a substance. A World Wide Web (WWW) server was used for the on-line prediction of the biological activity [17]. The potential bioactivities of the $\mathrm{H}^{+} / \mathrm{K}^{+}$-ATPase inhibitor were predicted by the PASS software product and the data of the prediction model were from Shanghai Institute of Organic Chemistry (SIOC).

\section{Conclusions}

A total of twelve metabolites of ilaprazole were detected in rat plasma after oral administration. The metabolites can be divided into three structural types: reductive metabolites with sulfide (M1, M3, M4, M5, and M10), oxidative metabolites with sulfone (M2, M9, M11, and M12), and hydroxylated metabolites with sulfoxide (M6, M7, and M8). The structures of the six new in vivo metabolites (M3, M7, M8, M9, M11, and M12) were identified and characterized via LC-HRMS and LC-MS ${ }^{\mathrm{n}}$. The fragmentation behaviors of ilaprazole and its metabolites were concluded as follows. The characteristic fragments of ilaprazole and its metabolites were created after the fragmentation of the bond between $\mathrm{C}$ and S. The typical fragment ion losing HS.(33 Da) would form in the metabolites with the structure of sulfide. The metabolites with the structure of sulfone would produce a characteristic fragmentation behavior with a neutral loss of $\mathrm{SO}_{2}(64 \mathrm{Da})$. The fragmentation behaviors of ilaprazole and its metabolites were described in this research, which could be valuable in rapidly detecting the metabolism of similar structures. The results of the in silico prediction suggested that all the new ilaprazole metabolites have shown the potential ability to inhibit $\mathrm{H}^{+} / \mathrm{K}^{+}$-ATPase activity. This provides stronger support for the efficacy of ilaprazole and gives an important basis for the design of new PPIs.

Supplementary Materials: The following are available online, Table S1: Accurate mass data of ilaprazole and twelve metabolites. Table S2: The possible structure of the metabolites. Figure S1A: The MSn spectra of Ilaprazole from $[\mathrm{M}+\mathrm{H}]^{+}$at $m / z 367$; Figure S1B: The MSn spectrum of $m / z 168$ ion from $[\mathrm{M}+\mathrm{H}]^{+}$at $m / z 351$ of M1; Figure S1C: The MSn spectrum of $m / z 200$ ion from $[\mathrm{M}+\mathrm{H}]^{+}$at $m / z 383$ of M2; Figure S1D: The MS ${ }^{n}$ spectra of M3 from $[\mathrm{M}+\mathrm{H}]^{+}$at $m / z$ 343; Figure S1E: The MS spectra of M4 from $[\mathrm{M}+\mathrm{H}]^{+}$at $m / z$ 367; Figure S1F: The $\mathrm{MS}^{\mathrm{n}}$ spectra of M5 from $[\mathrm{M}+\mathrm{H}]^{+}$at $m / z$ 383; Figure S1G: The MS ${ }^{\mathrm{n}}$ spectra of M6 from $[\mathrm{M}+\mathrm{H}]^{+}$at $m / z 383$; Figure S1H: The MS ${ }^{\mathrm{n}}$ spectra of M7 from $[\mathrm{M}+\mathrm{H}]^{+}$at $m / z$ 383; Figure S1I: The $\mathrm{MS}^{n}$ spectra of M8 from $[\mathrm{M}+\mathrm{H}]^{+}$at $m / z$ 399; Figure S1J: The MS ${ }^{n}$ spectra of M9 from $[\mathrm{M}+\mathrm{H}]^{+}$at $m / z$ 399; Figure S1K: The MS ${ }^{n}$ spectra of M10 from $[\mathrm{M}+\mathrm{H}]^{+}$at $m / z$ 399; Figure S1L: The $\mathrm{MS}^{n}$ spectrum of $m / z 381$ ion from $[\mathrm{M}+\mathrm{H}]^{+}$at $m / z 399$ of M11; Figure S1M: The MS ${ }^{n}$ spectrum of $m / z 216$ ion from $[\mathrm{M}+\mathrm{H}]^{+}$at $m / z 399$ of M12. Figure S2A: The HRMS/MS spectrum and the possible fragmentation pathway of M4; Figure S2B: The HRMS/MS spectrum and the possible fragmentation pathway of M5; Figure S2C: The HRMS/MS spectrum and the possible fragmentation pathway of M6; Figure S2D: The HRMS/MS spectrum and the possible fragmentation pathway of M10.

Author Contributions: Conceptualization, methodology and writing-original draft preparation, G.Z.; interpretation of the data and revision of the manuscript, K.G.; investigation and research, P.W. 
and Y.S.; methodology, supervision and review and revision of the manuscript, C.M. All authors have read and agreed to the published version of the manuscript.

Funding: This research received no external funding.

Institutional Review Board Statement: The study was approved by The Animal Care and Welfare Committee of Institute of Materia Medica, Chinese Academy of Medical Sciences and Peking Union Medical College. The protocol code was 00005960. It has been approved on 9 January 2020.

Informed Consent Statement: Not applicable.

Data Availability Statement: The data presented in this study are available on request from the corresponding author.

Acknowledgments: The authors are thankful to the Shanghai Institute of Organic Chemistry, Chinese Academy of Sciences, for their support with the in silico analysis.

Conflicts of Interest: The authors declare no conflict of interests.

Sample Availability: Not available.

\section{References}

1. Savarino, E.; Ottonello, A.; Martinucci, I.; Dulbecco, P.; Savarino, V. Ilaprazole for the treatment of gastro-esophageal reflux. Expert Opin. Pharmacother. 2016, 17, 2107-2113. [CrossRef] [PubMed]

2. $\quad$ Bang, C.S.; Shin, W.G.; Seo, S.I.; Choi, M.H.; Jang, H.J.; Park, S.W.; Kae, S.H.; Yang, Y.J.; Shin, S.P.; Baik, G.H.; et al. Effect of ilaprazole on the healing of endoscopic submucosal dissection-induced gastric ulcer: Randomized-controlled, multicenter study. Surg. Endosc. 2019, 33, 1376-1385. [CrossRef] [PubMed]

3. Liao, X.-M.; Nong, G.-H.; Chen, M.-Z.; Huang, X.-P.; Cong, Y.-Y.; Huang, Y.-Y.; Wu, B.-H.; Wei, J.-Q. Modified sequential therapy vs. quadruple therapy as initial therapy in patients with Helicobacter infection. World J. Gastroenterol. 2015, 21, 6310-6316. [CrossRef] [PubMed]

4. Xuan, J.; Song, R.L.; Xu, G.X.; Lu, W.Q.; Lu, Y.J.; Liu, Z. Modeling the cost-effectiveness of ilaprazole versus omeprazole for the treatment of newly diagnosed duodenal ulcer patients in China. J. Med Econ. 2016, 19, 1056-1060. [CrossRef] [PubMed]

5. Kwon, D.; Chae, J.; Park, C.; Kim, Y.; Lee, S.; Kim, E.; Huh, I.; Kim, N.; Cho, K. Effects of IY-81149, a Newly Developed Proton Pump Inhibitor, on Gastric Acid Secretion in vitro and in vivo. Arzneimittelforschung 2011, 51, 204-213. [CrossRef] [PubMed]

6. Ji, X.-Q.; Du, J.-F.; Chen, G.; Chen, G.; Yu, B. Efficacy of ilaprazole in the treatment of duodenal ulcers: A meta-analysis. World J. Gastroenterol. 2014, 20, 5119-5123. [CrossRef] [PubMed]

7. Shin, J.S.; Lee, J.Y.; Cho, K.-H.; Park, H.L.; Kukulka, M.; Wu, J.-T.; Kim, D.Y.; Park, S.-H. The pharmacokinetics, pharmacodynamics and safety of oral doses of ilaprazole 10, 20 and $40 \mathrm{mg}$ and esomeprazole $40 \mathrm{mg}$ in healthy subjects: A randomised, open-label crossover study. Aliment. Pharmacol. Ther. 2014, 40, 548-561. [CrossRef] [PubMed]

8. He, C.; Wan, H. Drug metabolism and metabolite safety assessment in drug discovery and development. Expert Opin. Drug Metab. Toxicol. 2018, 14, 1071-1085. [CrossRef] [PubMed]

9. Seo, K.-A.; Lee, S.-J.; Kim, K.-B.; Bae, S.K.; Liu, K.-H.; Kim, D.-H.; Shin, J.-G. Ilaprazole, a new proton pump inhibitor, is primarily metabolized to ilaprazole sulfone by CYP3A4 and 3A5. Xenobiotica 2012, 42, 278-284. [CrossRef] [PubMed]

10. Li, Y.; Zhang, W.; Guo, N.; Zhou, G.; Zhou, H.; Xiao, Z. Pharmacokinetics of the new proton pump inhibitor ilaprazole in Chinese healthy subjects in relation to CYP3A5 and CYP2C19 genotypes. Clin. Chim. Acta 2008, 391, 60-67. [CrossRef] [PubMed]

11. Myung, S.-W.; Min, H.-K.; Jin, C.; Kim, M.; Lee, S.M.; Chung, G.J.; Park, S.J.; Kim, D.Y.; Cho, H.-W. Identification of IY81149 and its metabolites in the rat plasma using the on-line HPLC/ESI mass spectrometry. Arch. Pharmacal Res. 1999, 22, 189-193. [CrossRef] [PubMed]

12. Zhou, G.; Tan, Z.-R.; Zhang, W.; Ou-Yang, D.-S.; Chen, Y.; Guo, N.; Liu, Y.-Z.; Fan, L.; Deng, H.-W. An improved LC-MS/MS method for quantitative determination of ilaprazole and its metabolites in human plasma and its application to a pharmacokinetic study. Acta Pharmacol. Sin. 2009, 30, 1330-1336. [CrossRef] [PubMed]

13. Zhou, G.; Shi, S.; Zhang, W.; Tan, Z.; Chen, Y.; Guo, N.; Zhou, H.; Hu, H.; Tan, J. Identification of ilaprazole metabolites in human urine by HPLC-ESI-MS/MS and HPLC-NMR experiments. Biomed. Chromatogr. 2010, 24, 1130-1135. [CrossRef] [PubMed]

14. Pu, J.; Wang, F.; Tang, W.; Zhu, M. Biotransformation of Ilaprazole in Human Liver Microsomes and Human: Role of CYP3A4 in Ilaprazole Clearance and Drug-Drug Interaction. Drug Metab. Dispos. 2018, 46, 1453-1461. [CrossRef] [PubMed]

15. Beccaria, M.; Cabooter, D. Current developments in LC-MS for pharmaceutical analysis. Analyst 2020, 145, 1129-1157. [CrossRef] [PubMed]

16. Wu, S.; Zhang, Y.; Zhang, Z.; Song, R. Use of LC-QqQ-MS for the detection of emodin metabolites in rat bile and urine. Biomed. Chromatogr. 2017, 31, e3979. [CrossRef] [PubMed]

17. Lagunin, A.; Stepanchikova, A.; Filimonov, D.; Poroikov, V. PASS: Prediction of activity spectra for biologically active substances. Bioinformatics 2000, 16, 747-748. [CrossRef] [PubMed] 
18. Zhong, D.; Xie, Z.; Chen, X. Metabolism of pantoprazole involving conjugation with glutathione in rats. J. Pharm. Pharmacol. 2005, 57, 341-349. [CrossRef] [PubMed]

19. Pearce, C.M.; Lushnikova, M.V. Microbiological production of omeprazole metabolites by Cunninghamella elegans. J. Mol. Catal. B Enzym. 2006, 41, 87-91. [CrossRef] 